\title{
3D Wayfinding Choremes: A Cognitively Motivated Representation of Route Junctions in Virtual Environments
}

\author{
Tassilo Glander ${ }^{1}$, Denise Peters ${ }^{2}$, Matthias Trapp ${ }^{1}$, Jürgen Döllner ${ }^{1}$ \\ ${ }^{1)}$ Hasso-Plattner-Institute, University of Potsdam, \\ Prof.-Dr.-Helmert-Strasse 2-3, 14482 Potsdam, Germany \\ [tassilo.glander, matthias.trapp, juergen.doellner]@hpi.uni-potsdam.de \\ ${ }^{2)}$ Transregional Collaborative Research Center SFB/TR 8 Spatial Cogni- \\ tion, University of Bremen \\ P.O. Box 330 440, 28334 Bremen, Germany \\ peters@sfbtr8.uni-bremen.de
}

\begin{abstract}
Research in cognitive sciences suggests that orientation and navigation along routes can be improved if the graphical representation is aligned with the user's mental concepts of a route. In this paper, we analyze an existing 2D schematization approach called wayfinding choremes and present an implementation for virtual 3D urban models, transferring the approach to 3D. To create the virtual environment, we transform the junctions of a route defined for a given road network to comply with the eight sector model, that is, outgoing legs of a junction are slightly rotated to align with prototypical directions in $45^{\circ}$ increments. Then, the adapted road network is decomposed into polygonal block cells, the individual polygons are extruded to blocks and their façades are textured. For the evaluation of our 3D wayfinding choreme implementation, we present an
\end{abstract}


experiment framework that allows to train and test subjects by route learning tasks. The experiment framework can be parameterized flexibly, exposing parameters to the conductor. We finally give a sketch of a user study by identifying hypotheses, indicators, and, hence, experiments to be done.

\section{Introduction}

The spreading applications of 3D geovirtual environments show that they are evolving from expert tools for selected applications to platforms addressing everyday needs of average users. For example, virtual 3D city models are used as integration platforms for real estate search, vacation planning, and traffic information. Thus, they serve as a spatial index structure for users to access data from different domains, revealing underlying spatial relations in the data.

However, in order to compete with 2D maps for navigation and orientation tasks, applications using 3D geovirtual environments need to provide additional benefits that legitimate the higher effort of creating and maintaining the used models, providing the infrastructure, such as a PC or a mobile device, and actually interacting with them. Given the higher complexity as one reason, many people have problems with navigation and orientation in virtual environments. For example, the acquisition of survey knowledge is difficult, as the impression from egocentric perspective has to be transferred to an allocentric model of the situation (Nash et al. 2000).

\subsection{Virtual Environments}

Viewed from a cognition science point of view, a virtual environment (VE) "[...] offers the user a more naturalistic medium in which to acquire spatial information, and potentially allows to devote less cognitive effort to learning spatial information than by maps.“ (Montello et al. 2004, pp:275).

Encouraged by this argument, lots of research has been done regarding navigation in virtual VEs. As interactive, real-time, 3D graphical renderings of spatial data, VEs are controlled by the users, thus directly changing and responding to users' behavior, e.g., through joystick or mouse interaction. In a VE, users typically have a dynamic first-person perspective on the scene. They acquire information sequentially and have to integrate them over time to build up a mental representation of the environment. Many studies show that people are able to learn spatial information from a VE (Montello et al. 2004). 
There is a long tradition to present spatial information in a static pictorial way, e.g., in maps, and using verbal descriptions. Several approaches aim at easing the use of navigation assistance systems to increase user's performance in navigation through an environment. Most of these approaches apply principles of information reduction or abstraction to the spatial data to emphasize the necessary information elements while hiding unnecessary or disturbing parts.

One of these approaches are the wayfinding choremes introduced by (Klippel et al. 2005). Choreme is coined from the greek word for space, chor, and the suffix -eme, and means elementary primitives of space in analogy to phonemes for speech, or graphemes for written language. These wayfinding choremes represent prototypes of turning actions, which can also be used to emphasize turning actions in wayfinding maps (Klippel et al. 2005).

In this paper, we transfer the schematization principles of wayfinding choremes, i.e., the concept of cognitively adequate route representations developed for maps, to 3D VEs (Figure 1). We want to investigate, if assumptions made for 2D plans work in 3D visualization in spite of the fact, that space reception is fundamentally different. After the introduction of the theoretical basis of 3D wayfinding choremes and a sketch of the experiment to be done, we will present an implementation of wayfinding choremes in a 3D VE, followed by our experiment application. Then, we will discuss the concept as well as relate our solution to other approaches. We will conclude this paper with an outlook.

This paper focuses on the concept of 3D wayfinding choremes, their technical realization and the implementation of an experiment application. We expect to get first results of our ongoing empirical studies in the next months.

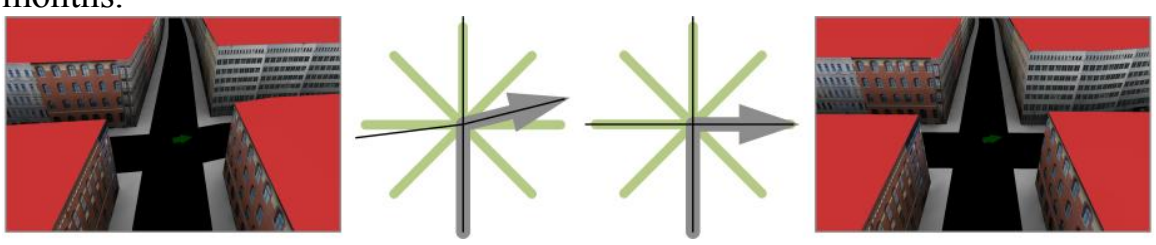

Fig. 1. Transformation of a junction into a prototypic configuration: The original roads (left) are analyzed, sorted and processed according to their angle to create a cognitively adequate representation of the junction (right). 


\section{3D Wayfinding Choremes}

When VEs are used for tourist information, the most important purpose is communication of knowledge of the environment to users, i.e., tourists, to enable them to navigate in the VE and/or to learn a route. Unfortunately, navigation is often difficult in VEs even for trained users, especially if they have to navigate in large environments. The difficulties arise from users' problems to orientate themselves in VEs; also the acquisition of survey knowledge of the environment is poorer compared to navigation in the real world. These problems to some extent originate from the absence of vestibular and proprioceptive stimuli; though, many difficulties and spatial behavior issues within virtual environment are only fragmentary understood (Nash et al. 2000).

There are different approaches aiming at improving users' navigation performance in VEs, for example, providing a map or positioning additional landmarks within the VE, e.g., (Darken and Sibert 1993, 1996), or redesigning structural elements of VEs founded by the theory of Lynch (1960), e.g., (Omer et al. 2006). So far, these studies present a large range of different setting in terms of subjects' exposure time, their level of expertise and also in the details of the represented VE, such as size and naturalism of representation, e.g., (Goerger et al. 1998; Richardson 1999). Hence, existing approaches have largely contradicting navigation performance results.

\subsection{Schematization}

In our approach, we decide to apply the principle of schematization for enhancing the structural information of our urban VE. Definitions of the term schematization strongly vary between science disciplines. In cognitive sciences, especially linguistics, schematization is interpreted in the context of information processing. Herskovit (1998) postulates that three distinguishable processes are involved in schematization: abstraction, idealization, and selection. In computer science and artificial intelligence, schematic representation focuses on the identification and extraction of information that is relevant for a task (Berendt et al. 1998). Hereby three types of knowledge are defined: Knowledge that is essential and therefore needs to be represented unaltered; knowledge that can be altered, but has to 

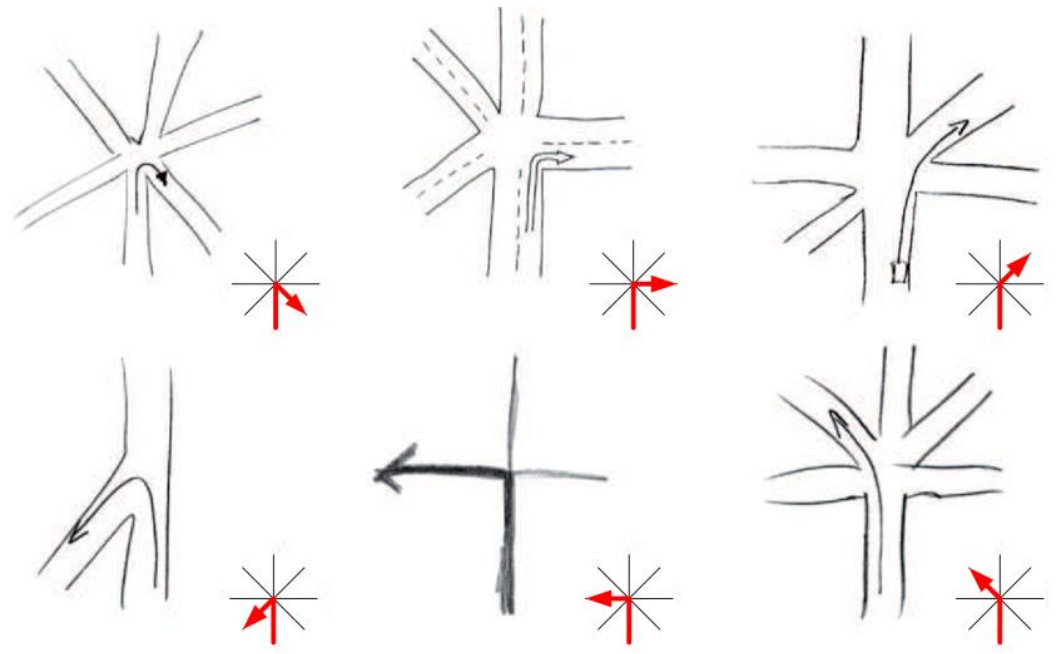

Fig. 2. Sketches of intersections with turning descriptions and their prototypes in the bottom right. (adapted from Klippel et al.( 2005))

be presented; and knowledge that should be omitted (Goerger et al. 1998; Palmer 1978). We understand schematization beyond the named definition as a process of intentionally simplifying a representation beyond technical needs to achieve cognitive adequacy, as defined in (Klippel et al. 2005).

Cognitive adequacy, defined by Strube (1992), means on the one hand the representation of cognitive processes or on the other hand representations that have the quality to support cognitive processes. Compared to generalization, as understood in cartography, schematization is more than a simplified representation - it is a cognitively motivated representation.

\subsection{D Wayfinding Choremes}

Representing spatial information in a two-dimensional way as maps has a long tradition. Usually, cartographic maps depict selected aspects of the environment on a spatial scale much smaller than 1:1. Schematization is one way of selecting the represented aspects beyond the technical need towards a cognitively motivated representation. Schematization principles are applied to improve the legibility of maps depending on the specific tasks. 

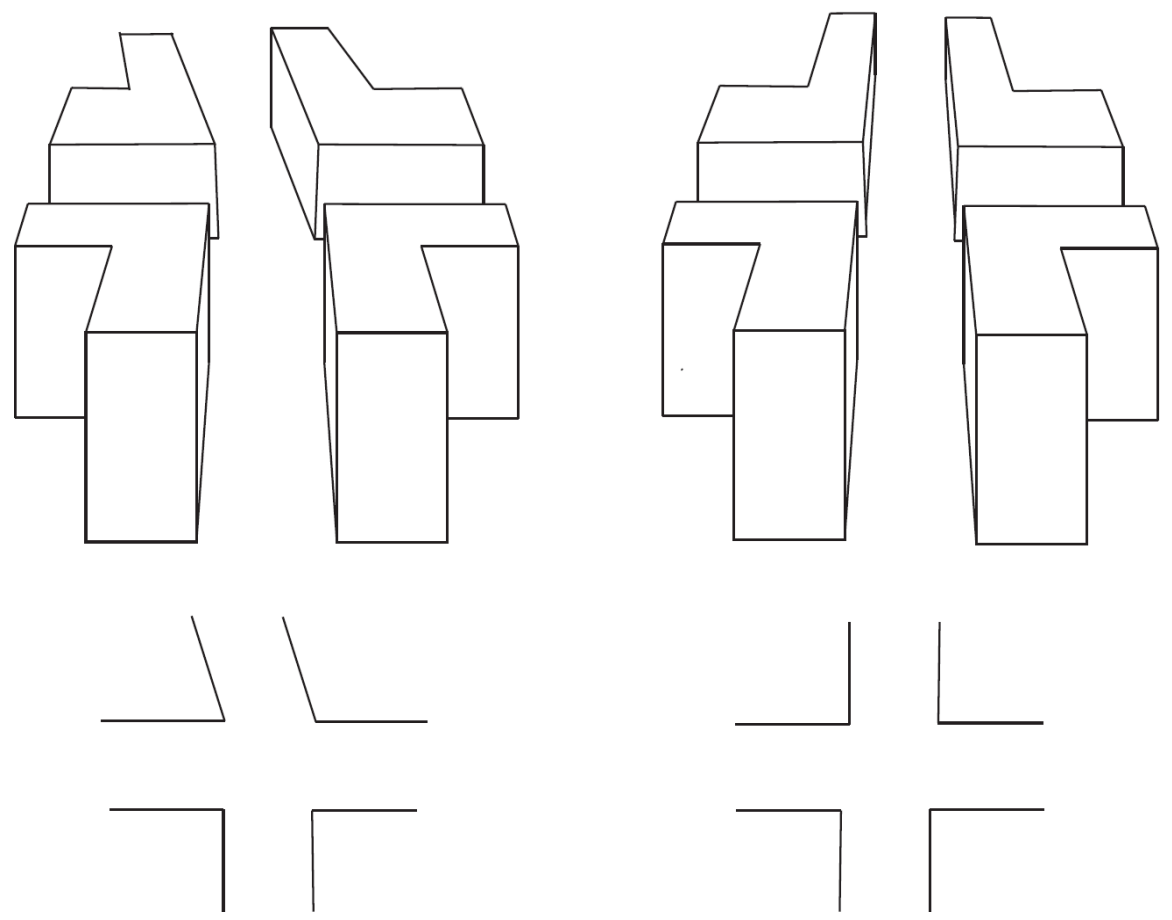

Fig. 3. A sketch of $3 \mathrm{D}$ wayfinding choreme. The original intersection (left) is compared with the prototype of this intersection (right). On the lower part, the respective 2D representation is shown. (from Peters and Richter (2008))

One example for cognitively adequate schematization principles for wayfinding maps are wayfinding choremes introduced by (Klippel et al. 2005). They define wayfinding choremes as mental conceptualization of functional wayfinding primitives. This schematization principle is aimed at easing the decision process at a junction by replacing the original curve by prototypes. Klippel et al. empirically identified mental conceptualizations of turning situations. Figure 2 shows sketches of resulting prototypical turning directions and the correlated wayfinding choremes. Participants tend to represent a turning action in a prototypical way.

Wayfinding choremes are prototypes of turning actions. Externalized graphically, they are useful to emphasize a turning action in a wayfinding situation. This method can be applied to route-following maps. Empirical data (Meilinger et al. 2007) show that schematic maps with prototypical junctions improve navigation performance for wayfinding compared to normal, that is, unchanged floor plans. 


\subsection{Transferring wayfinding choremes to 3D}

In our approach, we apply the schematization principle of wayfinding choremes to VEs. As explained in the beginning, navigation in VEs is a difficult navigation task. For enhancing navigation performance, 3D wayfinding choremes have been suggested (Peters and Richter 2008), applying the principle of wayfinding choremes to 3D representations by locally changing the angular configuration of road junctions. Furthermore, not only angles of the intersecting roads but also angles of buildings have to be changed locally at the intersection, as sketched in Figure 3. For the implementation of $3 \mathrm{D}$ wayfinding choremes, it is not necessary to change the global configuration of the street network. We will explain our implementation in detail in Section 3.

"Much of the knowledge about time and space is qualitative in nature. Specifically, this is true for visual knowledge about space." (Freksa 1991, pp:365). In our approach, we aim to emphasize the qualitative nature of VEs by applying the schematization principle of wayfinding choremes. By replacing junctions with prototypes, this schematization approach eases the decision for actions to be performed at these intersections, as a displayed prototypical direction instead of the real angle (e.g., $90^{\circ}$ instead of $79.3^{\circ}$ ) can be understood easily. This can be argued, as the estimation of angles is a difficult task in VEs (Riecke 2003). It can be expected that orientation is more accurate using prototypical angles.

\subsection{Experiment}

To define requirements for software that creates a VE and supports conducting experiments to evaluate the wayfinding choreme concept in 3D, we give a sketch of an experiment setup. We hypothesize that 3D wayfinding choremes in VEs improve navigation performance. Therefore, we will compare route learning performance in an unchanged VE with the transformed VE. The subjects will learn the route by actively navigating through the VE, following the indicated route that is highlighted by arrows. Then, the participants have to navigate along the route without additional route indicators being presented. We will analyze the number of errors of the participants when reproducing the route as a measure for their performance. An error is defined as a wrong turning decision that is not recognized within a predefined distance to the route. The subjects will be divided in two groups: One group will learn the route in an unchanged world and the other one in a choremized world. 
Additionally, we will perform some pre-testing of the perceptual and also qualitative nature of intersections in VEs. We will analyze the quality of estimation of angles in the VE by the subjects and let them draw sketch maps.

\section{Creation of a Choremized World}

Targeting the evaluation of the application of the wayfinding choreme concept in a user study, we design an experiment framework as follows (Figure 4): We need two main components, the world creation component to derive the VE using a given set of input data under certain parameters, and the interactive viewer application that helps conducting the experiment.

In the following, we will firstly describe the creation of the VE, and then present the application (Section 4).

\subsection{Design Decisions}

The VE has to look sufficiently similar to a real urban situation to be sufficiently convincing to the subjects. For the evaluation, three requirements exist to allow studying the impact of choreme transformation of junctions in the context of route navigation:

- The use of landmarks for orientation needs to be controlled and limited.

- The junctions and the course of the roads have to be emphasized.

- The terrain is planar as the concept does not cover implications for 3D terrain.

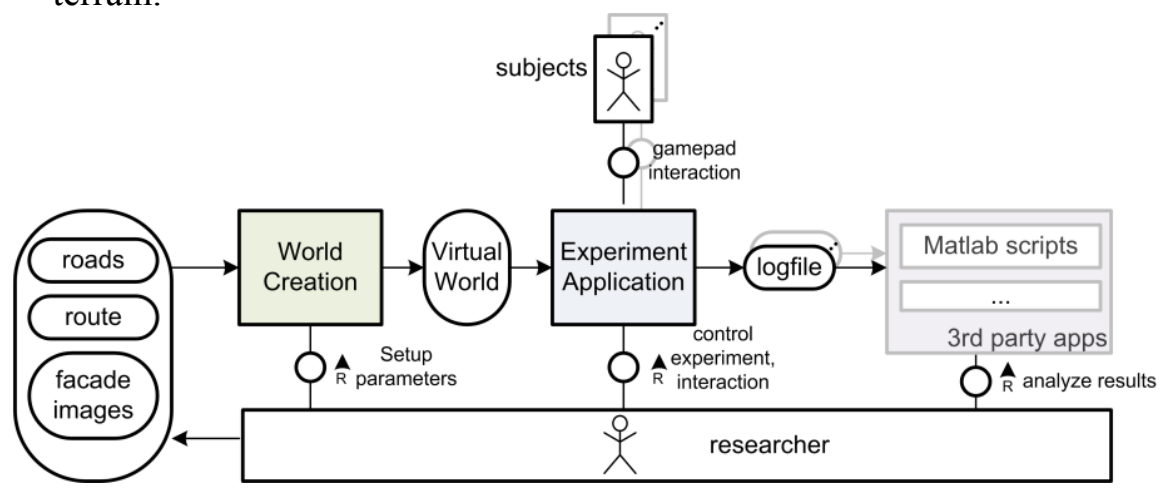

Fig. 4. Overview of the experiment framework. 

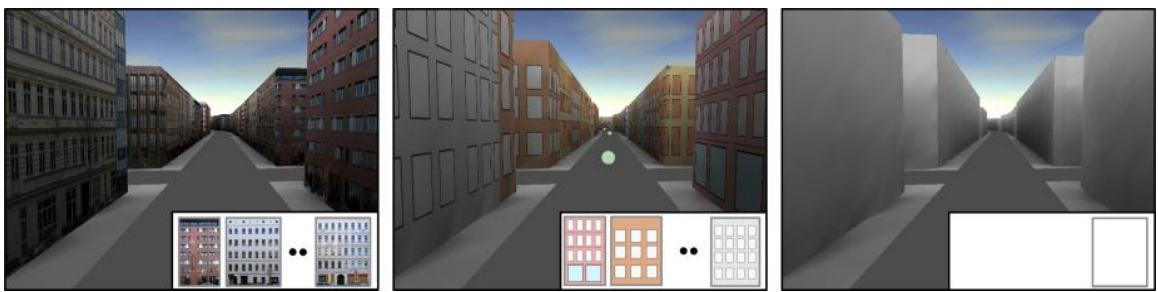

Fig. 5. Façades can be visualized naturalistic, hand-drawn, or white.

Therefore, the complexity of the VE's visualization has to be reduced to limit the number of potential landmarks. To emphasize the course of roads and junction configurations, building façades are aligned with the roads. Additionally, explicit street polygons are embedded in the scene.

The creation of the choremized world can be sketched as follows. First, the input road network is processed using the input route to transform the route junctions to follow the choreme concept. The resulting transformed road network is then used to create polygonal partitions, i.e., cells. These cells are then extruded to yield 3D blocks.

\subsection{Input Data}

A virtual 3D city model typically integrates data from various sources and of different types, such as building models, infrastructure networks, digital terrain models, terrain textures, city furniture positions and models, and vegetation models (Kolbe et al. 2005). For our exemplary urban environment, we use just a subset of those to create an urban environment sufficient to visualize the choreme aspect along a route.

First of all we need a road network, which is in our case a part of a real world city. Data providers are TeleAtlas or OpenStreetMap, but also a manually created set of roads can be used. Our application reads them as line features from a Shapefile. The route also needs to be given as a Shapefile with a single line feature that is approximately aligned with the road network.

By creating urban VEs, we need for the building façades a set of façade images that are sufficiently generic, that is, they do not contain overly distinctive features. Using individual, remarkable façades would allow the subjects to use them as landmarks, which we want to control. In our scenario, we use a selected set of 38 different façade photos. Alternatively, completely synthetically façade images can be used, or no façade images at all (Figure 5). 
Since we want to enable the controllable integration of landmark objects, we allow specifying landmark positions in a Shapefile along with a set of 3D models from typical city furniture objects. On each point in the Shapefile, one of the 3D models is placed as a remarkable landmark object on the streets.

\subsection{Route Processing}

\subsubsection{Deriving Topology}

Once the road network and the route are read in, the road network is converted into an arrangement (Wein et al. 2007; de Berg et al. 2008), which basically intersects all line features $L=\left\{l_{i}\right\}$ to create a half-edge data structure $G=(V, E, P)$ with vertices $V$, edges $E$, and polygons $P$ with the usual operations (de Berg et al. 2008).

$$
L=\left\{l_{i}\right\} \stackrel{\text { arrangement }}{\longrightarrow} G=(V, E, P)
$$

The half-edge data structure is necessary to do further processing that also regards the topology, e.g., does consider neighbor relations of junctions. In addition, it allows querying the polygonal decomposition $P$ of the plane that is implicated by the set of linear features.

As the topological graph structure on the road network does not exist at the time of the route specification, the geometric route feature $r=\left\{r_{1}, \ldots, r_{n}\right\}$ with $r_{i}=(x, y), x, y \in R$ has to be aligned with and mapped to the topological road network representation. This is done by searching for geometrically near and connected vertices of the computed half-edge structure.

$$
(G, r) \stackrel{\text { alignment }}{\longrightarrow} r_{G}=\left\{v_{1}^{r}, \ldots, v_{m}^{r}\right\} \quad v_{j}^{r} \in V
$$

At the end, a topological representation of the route has been derived, e.g., an ordered list of connected nodes in the graph structure.

\subsubsection{Applying Choreme Transformation}

The graph together with the route information is then used to apply the geometric transformation to junctions along the route, i.e., nodes of degree greater than two. It is therefore a transformation of the half-edge structure depending on the route, $\left(G, r_{G}\right) \stackrel{\text { chorematiztion }}{\longrightarrow} G^{\prime}$. Note that the direction of the route is important, as, considering a single junction, the incoming road has to be kept unchanged, while all other outgoing roads have to be 


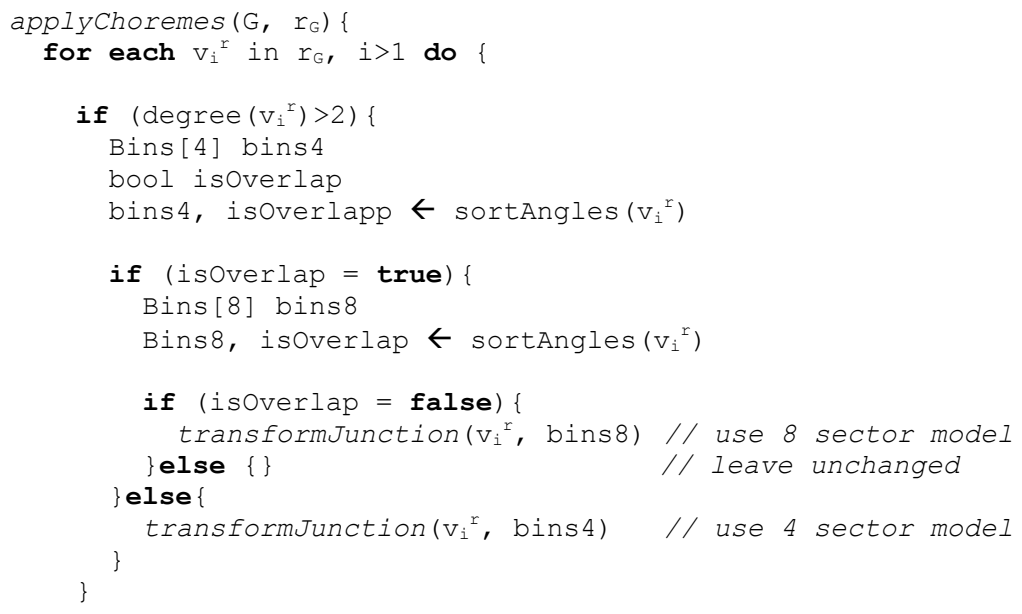

Fig. 6. Pseudo-code for creating a choreme representation.

changed to conform to the direction model (Klippel 2005). Hence, the algorithm iterates through all nodes along the route, starting with the first node and transforming all junctions to the destination node (Figure 6).

For one junction, the existing outgoing road segments are at first evaluated to compute the angles relative to the incoming road segment. Using the graph structure, neighboring edges can easily be queried for a certain vertex, hence we get positions of the neighboring vertices for the computation. The result is a numerical representation of the junction, containing the angles of each outgoing leg. These are sorted into bins with a defined size, first, into $90^{\circ}$-sized bins, i.e., a four-sector model, and then, in case of multiple legs within one bin, into $45^{\circ}$-sized bins. If there are still multiple items in one bin after the second iteration, the junction is not changed, as it can be argued that the junction is too specific for a generalization.

In the next step, the outgoing road segments are rotated slightly to follow the prototypic directions (Figure 7). This has a number of implications: As the transformation effect should be sufficiently local, i.e., within a radius $r$, and other junctions' positions are to be kept fix, new vertices have to be inserted along the changed edge. The prototypical direction should be remarkable, though it is not clear what this means in terms of meters. Therefore, our algorithm exposes the desired length of the prototypical direction as a parameter, being $r / 2$. In case this length cannot be guaranteed, as another junction is within the desired length as shown in Figure 7, we insert the point half-way to the next junction. The connection 


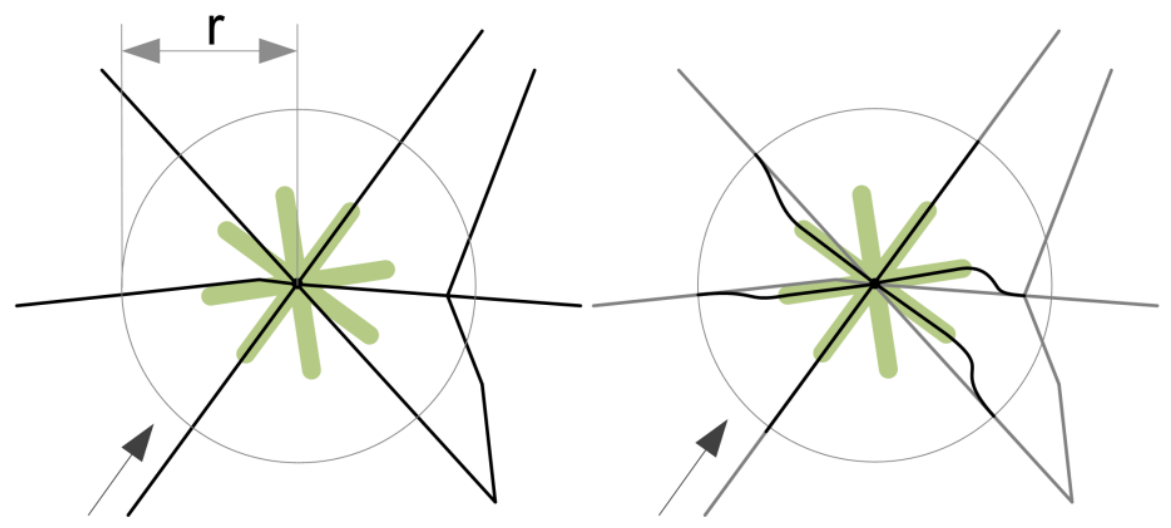

Fig. 7. The transformation applied to a junction. The prototypic directions are superimposed, the gray circle showing the local limitation of the effect. The shifted roads are smoothed using Beziér curves (right) when connected with the subsequent original edge.

of the inserted and shifted vertex with the original road network is then, as in the original paper, smoothed by applying a Bézier curve (Klippel et al. 2005). The algorithm stops, when all junctions along the route have been processed.

\subsection{Creation of the 3D Geometry}

After the 2D processing, 3D geometry has to be created for an explorable VE. Therefore, the half-edge data structure is queried for the polygons, which need to be further processed to be the footprints for block cells. At first, using 2D Boolean operations, the road network is subtracted from the polygons, cutting away space for the roads.

Then, the footprints are extruded to typical urban building heights, which can be given as an interval specifying minimum and maximum height. For an individual block, the height is then randomly varied within the interval, and for again randomly sized façade sections, textures from the façade textures pool are assigned (Figure 8). The texture parameterization is stored, so that we can apply approximately the same parameterization to both the choremized and the unchanged version of the world.

The resulting urban-like building blocks are finally pre-shaded and stored together with collision geometry necessary for collision free navigation, later. 


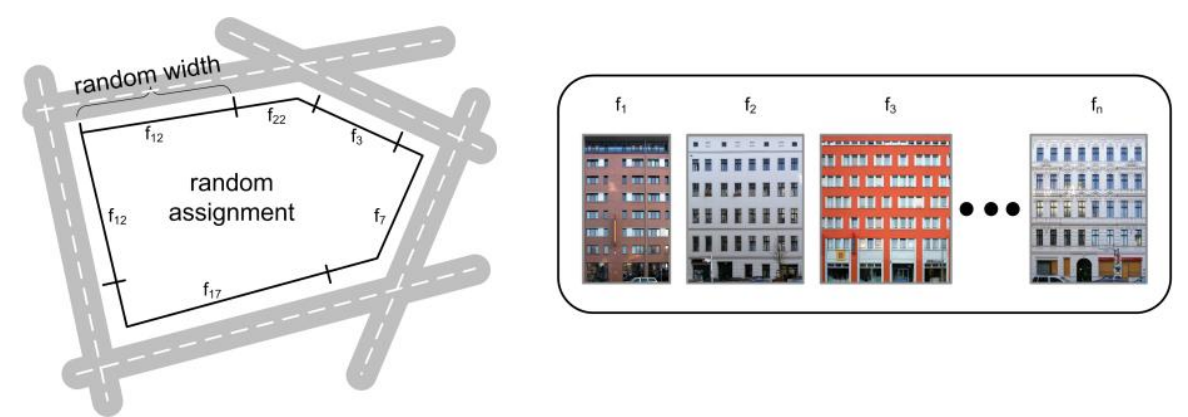

Fig. 8. A cell block's façade parameterization. A block, which is defined by its surrounding streets, is randomly textured from a pool of given façade textures.

\subsection{Computational Complexity}

The computation of the choreme representation starting from the road network and the route depends linearly on the size of those two data components. As the algorithm to change route junctions iteratively goes through all junctions along the route, and for each junction only the topological neighborhood is processed, the complexity is $O(n)$. The most expensive operations are done after the choreme transformation for the creation of the virtual environment, i.e., the Boolean operations and the lighting.

For an overview of the processing timings, we look at two data sets of differently sized road network and two routes drawn above them.

Small World The small data set covers an area of $1500 \times 2000 \mathrm{~m}^{2}$ and contains 722 line features with 2330 points.

Large World The larger data set of the two covers $3000 \times 2700 \mathrm{~m}^{2}$ and contains 1730 line features with 5450 points.

Short Route The short route has 8 junctions on a length of $1660 \mathrm{~m}$.

Long Route The long route has 51 junctions on a length of $4300 \mathrm{~m}$.

The Small World data set is a subset of the Large World data set. Comparing the times (Table 1) to compute these different data sets reveals, that most of the time is spent with geometry creation. During the geometry creation phase, the polygons are trimmed through the surrounding streets by performing buffering and Boolean difference operations. Then, the resulting polygons are extruded and textured and collision geometry is created. However, the Boolean operations take the most time. 
Table 1. Timings for the computation of differently sized worlds.

\begin{tabular}{lccc}
\hline & $\begin{array}{c}\text { Small World } \\
\text { short route }[\mathrm{s}]\end{array}$ & $\begin{array}{c}\text { Large World } \\
\text { short route }[\mathrm{s}]\end{array}$ & $\begin{array}{c}\text { Large World } \\
\text { long route }[\mathrm{s}]\end{array}$ \\
\hline Choreme Transformation & 0.05 & 0.11 & 0.13 \\
Geometry Creation & 10.73 & 25.83 & 29.94 \\
Pre-Lighting & 6.03 & $9.61 \mathrm{~s}$ & 10.05 \\
\hline
\end{tabular}

\section{An Interactive Application for Experiments}

For evaluating the concept of applying wayfinding choremes on a route in a VE, we have created an interactive application. It allows the user to explore the VE with and without choreme transformation, thus allowing to compare both navigation performances. Furthermore a number of parameters of the visualization for the subject can be controlled. The application works as a tool to conduct and trace experiments with different subjects and different settings. In an experiment, the subjects' behavior, i.e., movement trajectories and heading information are stored in a log file together with name, date and the current visual parameter settings. Therefore it is suitable for arbitrary user studies targeting VEs that compare subjects' performance along a route in two different configurations of a scene.

The application is written in $\mathrm{C}++$ and uses the Virtual Rendering System (VRS) ${ }^{1}$ for $3 \mathrm{D}$ scene visualization and interaction, and Qt for the user interface.

\subsection{Exploration Mode}

After loading a data set, the application is in exploration mode, allowing free navigation in the VE. It is targeted at the experiment conductor to inspect the chosen route and the choreme effect. Therefore, the two representations of the world, that is, with and without the choreme transformation along the route, can be switched instantly. The user can also jump directly to junctions along the route for quick inspection.

A separate properties panel allows the configuration of the VE and experiment parameters independently from the main window showing the $3 \mathrm{D}$ scene. In addition to switching between the two world representations and loading another data set, the landmark objects and guiding arrows along the route can be disabled to compare the subjects' behavior (Figure 9). Especially, the field of view of the virtual camera can be configured to permit

${ }^{1}$ The Virtual Rendering System, www.vrs3d.org 


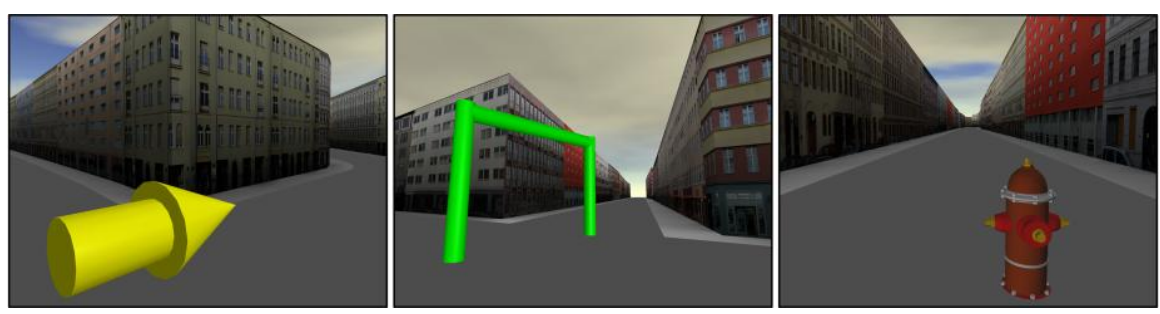

Fig. 9. The display of additional elements of the VE can be toggled, e.g., route indicating arrows (left) and the destination (center), as well as landmark objects (right).

the adaptation of the visualization to the experiment's setup. This is important, as it directly impacts humans' ability to estimate angles as well as the feeling of presence within the VE (Lin et al. 2002).

While the virtual environment can be explored freely using mouse and keyboard, for the experiment subjects, we chose a limited, gamepad-based interaction. As the navigation should be as simple and direct as possible, requiring the least possible learning effort, we decided to use an input device known to many from computer games. Further, the movement using the gamepad is restricted to a horizontal plane parallel to the virtual terrain at $1.80 \mathrm{~m}$ height. To keep the subjects from going behind the façades, a simple collision handling mechanism is applied.

\subsection{Experiment Mode}

During the experiment, the application guides and documents the process to support the conducting researcher. Depending on whether route arrows are displayed, we differentiate, if the subjects are to learn or to reproduce the route from memory.

When the researcher starts the experiment, the application sets the position to the beginning of the route and displays a start message. Then, the subject can move along the streets, possibly following the route until the destination is reached. The movement is limited by the route in that the subject is invisibly caged within a medium distance, e.g., $30 \mathrm{~m}$ around it.

The current status of the experiment, i.e., started, paused, finished, or canceled, can be controlled with the properties panel, or changes are triggered by user actions, i.e., when the destination is reached. The attempt of leaving the route is also communicated to the user by flashing red.

Most important for the experiment's analysis is the potential for automatic evaluation. Therefore, experiment relevant data is documented in a well defined ASCII file for later post-processing in tools such as Matlab. 
Each experiment is documented with a header containing a given subject identifier and the environment's configuration. The progress of the subject is tracked with respect to position and heading in regular, configurable time intervals, such as $1 / 10 \mathrm{~s}$. It is supplemented by status change events and collision events when the route is left. Thus, errors of the subjects while reproducing the route can be counted as an indicator for the adequacy of the representation.

\subsection{Experiment Questions}

Using our experiment application, a number of hypotheses can be evaluated both quantitatively and qualitatively.

Estimating Angles The estimation of angles of outgoing legs of a junction is the basis for a determination of the feasibility of the choreme approach in $3 \mathrm{D}$. The situation is fundamentally different compared to $2 \mathrm{D}$ maps, as the subjects have to transfer the egocentric impression from the $3 \mathrm{D}$ visualization to a mental allocentric concept of the junction. In our application, the subjects can be told to explore a junction and then asked to sketch a top-down view of it. We are interested, to which degree the estimation of the angles is correct.

Choreme Impact on 3D Route Comprehension While cognitively adequate representationof spatial data was shown to be effective for $2 \mathrm{D}$ floor plans (Meilinger et al. 2007), our application addresses the 3D case. It is possible to assess the choreme transformation of a 3D city environment along a route. The application automatically tracks attempts to leave the route, e.g., when a subject has forgotten the correct way. By counting these attempts, we have a quantitative measure for the confidence of the learned route, and, hence, can compare a subject's performance in the unchanged and the changed world.

Landmarks along the Route Landmarks undoubtedly play an essential role in the wayfinding process. Our application allows for the definition of landmark objects' positions and models to control their integration in the VE. Therefore, the subjects' wayfinding performance in different configurations can be measured and compared.

As the application only uses a small set of sufficiently generic, photorealistic or synthetic façades or even no façades at all, we can exclude as far as possible the effect of façades used as landmarks. In addition, the flat terrain and the simple scene structure with low detail ground complexity limit memorization of the route to the sequence of junctions and their configuration. 


\section{Discussion}

The presented framework for the creation of a virtual city environment externalizes the mental concept of a route and can be used in different settings. As our focus is on experiments evaluating the usefulness of $3 \mathrm{D}$ wayfinding choremes, the presented implementation creates a geometrically simple urban environment that still provides first insights into this question. In addition to using the experiment application in our own experiments, it is possible to export the created VEs for use in other applications in standard formats, e.g., CityGML (Kolbe et al. 2005) or VRML. Thus, the $3 \mathrm{D}$ environments can be evaluated by the community, or other experiments can be done. Another interesting opportunity is to use the transformed road network as input data for a procedural city model creation (Müller et al. 2006) as it would lead to much higher detailed buildings and ground level geometry to increase the photo-realistic impression. Still, the environment could be created in a controlled way.

\subsection{Concept}

Regarding the potential to transfer the wayfinding choreme concept to 3D, we are not sure, what the results of the experiments will be. The applicability of the concept in 3D is contradicted by our subjective impression that the changes of a junction's configuration are quite small. In addition, it is questionable, if the simplification of the route junctions in the sense of the choreme concept leads to a better comprehension and memorization of the route, or if it removes unique properties necessary for memorization. Finally, in our world 3D junctions are especially perceived through the closed façades. In a more photo-realistic 3D world, apart from very dense central city areas, buildings have free spaces between them, making perception and judgment of a junction more difficult. This effect does not occur on 2D maps, as roads are typically printed as lines and a possible transformation directly gets visible.

However, previous experiments have shown that route junctions are not remembered with exact angles regarding their configuration (Tversky and Lee 1999). Therefore, a simplification according to the wayfinding choremes reduces the presented information to the necessary aspects, thus easing the process of picking up the path. Therefore we are interested in conducting the experiment for $3 \mathrm{D}$ environments to create a more sound base for further discussion. 


\subsection{Comparison with Existing Work}

If we compare our work with existing approaches, routes are usually visualized using additional scene elements that give directional hints. Typically, line features aligned with the course of the route or arrows at decision points are integrated in the visualization to guide the user. These elements can be integrated, for example, within live video imagery as an overlay showing route indicators in a perspectively correct way (Narzt et al. 2004). In (Coors et al. 2005), multi-modal representations of routes are analyzed, including verbal instructions, 2D and 3D route hints. Our solution uses integrated elements during learn phase.

Current commercial solutions for navigation systems typically do rely on these explicit route indicators and integrate them in 2D views as well as, increasingly, 3D views. However, 3D visualization in navigation systems is in its infancy. Today's solutions just show annotated 3D models of the environment and use only few techniques to support perception and comprehension of the route.

In addition to explicit route indicators, a route and its environment can be visualized in a deformed way to ease its communication. The classical example for 2D route visualization, (Agrawala and Stolte 2001), simplifies the route by shrinking distances of straight route segments while preserving angles. In (Böttger et al. 2008), a metro plan, i.e., a schematic, topological route representation, is used to transform a topographic map accordingly with image warping techniques, revealing the spatial relations between the metro stations. For short routes within a 3D VE, (Degener et al. 2008) present an image warping technique to create a single image describing the way. Focus + context visualization as in (Trapp et al. 2008) is another way to support wayfinding along routes in 3D VEs. Our approach focuses on the geometric transformation of junctions to conform to the cognitive concept of a route.

The automatic creation of artificial virtual 3D city models is typically done using procedural techniques. Based on a grammar, that is, a set of rules, whole city models including a road network can be created (Parish and Müller 2001). This technique can also be extended to create different, complex building types (Müller et al. 2006). A similar approach with the focus on interactive road network creation is presented in (Kelly and McCabe 2007). As we create a VE aiming at the conduction of an experiment, we currently do not need a very high degree of realism. 


\section{Conclusion \& Outlook}

We present a framework to create a cognitively adequate representation of a route in a 3D virtual environment following the concept of wayfinding choremes, transferring the concept from 2D maps. The framework includes an application to conduct experiments for the evaluation of this transferred concept and support the conducting researcher with the automatic logging of relevant events during one experiment.

Our next steps will be the accomplishment of experiments, testing the memorization of a route in the unchanged and the transformed environment. In addition to monitor and flat-screen projector based tests, we plan to do the experiment in a half cylinder. We presume, that the results will be different compared to the flat screen experiment, as the immersion is enhanced, and, standing on a junction the subjects actually can look into the outgoing roads without further interaction. This will probably influence the ability to estimate angles, and, hence, the impact of the choreme transformation.

Technically, we plan to change our implementation in some aspects. An enhancement for the choreme concept refines the eight-sector-model and suggests a nonuniform size of the different sectors (Klippel et al. 2004). Especially aiming at verbal communication of directions, for example, the sector straight ahead is smaller compared to the directions "veer left" or "veer right". Another interesting idea is the dynamic adaptation of junctions as the user gets near, possibly using image warping techniques (Böttger et al. 2008). This would be needed for dynamic rerouting in case of a lost way and thus is relevant for car navigation systems.

Finally, the results of the experiments will eventually be integrated into the experiment application and/or be used in a refined concept.

\section{Acknowledgement}

We want to thank Sebastian Pasewaldt for supporting us with the implementation. We also thank the reviewers for their comments.

This work has been funded by the German Federal Ministry of Education and Research (BMBF) as part of the InnoProfile research group "3D Geoinformation" (www.3dgi.de).

This work is also supported by the Transregional Collaborative Research Center SFB/TR 8 Spatial Cognition, which is funded by the Deutsche Forschungsgemeinschaft (DFG). 


\section{References}

Agrawala, M. and Stolte, C. (2001) Rendering Effective Route Maps: Improving Usability Through Generalization, in: Proceedings of ACM SIGGRAPH, ACM press.

Berendt, B., Barkowsky, T., Freksa, C., and Kelter, S. (1998) Spatial Representation with Aspect Maps, in: LNCS: Spatial Cognition - An Interdisciplinary Approach to Representing and Processing Spatial Knowledge, Springer, pp. 313-336.

Böttger, J., Brandes, U., Deussen, O. and Ziezold, H. (2008) Map Warping for the Annotation of Metro Maps, in: IEEE Computer Graphics and Applications, 28(5), IEEE Computer Society Press, pp. 56-65.

Coors, V., Elting, C., Kray, C. and Laakso, K. (2005) Presenting Route Instructions on Mobile Devices: From Textual Directions to 3D Visualization, in Dykes, J., MacEachren, A.M. and Kraak, M.-J (eds.): Exploring Geovisualization, Elsevier, pp. 529-550.

Darken, R. P and Sibert, J. L. (1993) A Toolset for Navigation in Virtual Environments, in: UIST: Proceedings of the $6^{\text {th }}$ annual ACM Symposium on User Interface Software and Technology, ACM press, pp. 158-165

Darken, R. P. and Sibert, J. L (1996) Wayfinding Strategies and Behaviors in Large Virtual Worlds, in: Proceedings of the SIGCHI conference, pp. 142149.

De Berg, M., Cheong, O., and van Kreveld, M. (2008) Computational Geometry: Algorithms and Applications, Springer.

Degener, P., Schnabel, R., Schwartz, C. and Klein, R. (2008) Effective Visualization of Short Routes, in: IEEE Transactions on Visualization and Computer Graphics 14(6), IEEE Computer Society Press, pp. 1452-1458.

Freksa, F., (1991) Qualitative Spatial Reasoning, in Mark, D.M and Frank, A.U. (eds.): Cognitive and Linguistic Aspects of Geographic Space, Springer, pp. 361-372.

Freksa, F. (1999) Spatial Aspects of Task-Specific Wayfinding Maps, in: Gero, J.S. and Tversky, B. (eds.): Visual and Spatial Reasoning in Design, University of Sidney, Key Centre of Design Computing and Cognition, pp. 15-32

Goerger, S.R., Darken, R.P.,. Boyd, M.A, Gagnon, T.A., Liles, S.W., Sullivan, J.A., and Lawson, J.P (1998) Spatial Knowledge Acquisition from Maps and Virtual Environments in Complex Architectural Spaces, in: Colorado Springs US AirForce Academy (ed.): 16th Applied Behavioral Sciences Symposium, pp. 6-10.

Herskovits, A. (1998) Schematization, in Olivier, P. and Gapp, K.P (eds.): Representation and Processing of Spatial Expressions, Lawrence Erlbaum Assiociates, pp 149-162.

Kelly, G. and McCabe, H. (2007) Citygen: An Interactive System for Procedural City Generation, in: Proceedings of Fifth International Conference on Game Design and Technology, ACM press, pp. 8-16. 
Klippel, A., Dewey, C., Knauff, M., Richter, K.-F., Montello, D.R., C. Freksa, and Loeliger, E.-A. (2004) Direction Concepts in Wayfinding Assistance Systems, in Workshop on Artificial Intelligence in Mobile Systems (AIMS'04).

Klippel A., Richter, K.-F., Barkowsky, T. and Freksa, C. (2005) The Cognitive Reality of Schematic Maps, in: Meng, L., Zipf, A. and Reichenbacher T. (eds): Mapbased Mobile Services - Theories, Methods and Implementations, Springer, pp 57-74.

Kolbe, T. H., Gröger, G. and Plümer, L. (2005) CityGML Interoperable Access to 3D City Models, in van Oosterom, P., Zlatanova, S. and Fendel, E.M. (eds.) Proc. of the 1st International Symposium on Geoinformation for Disaster Management, Springer.

Lin, J.J.W., Duh, H.B.L., Parker, D.E., Abi-Rached, H. and Furness, T.A. (2002) Effects of Field of View on Presence, Enjoyment, Memory, and Simulator Sickness in a Virtual Environment, in: Proceedings of the IEEE Virtual Reality Conference, IEEE Computer Society press.

Lynch K. (1960) The Image of the City, MIT Press.

Meilinger, T., Hölscher, C., Büchner, S.J. and Brösamle, M. (2007) How Much Information Do You Need? Schematic Maps in Wayfinding and Self Localisation, in: LNCS: Spatial Cognition, Springer.

Montello, D. R., Hegarty, M. and Richardson, A. E (2004) Human Spatial Memory: Remembering Where, Chapter Spatial Memory of Real Environments, Virtual Environments, and Maps, Lawrence Erlbaum Associates, pp. 251285.

Müller, P., Wonka, P., Haegler, S., Ulmer, A. and van Gool, L. (2006) Procedural Modeling of Buildings, in: Proceedings of ACM SIGGRAPH 2006 / ACM Transactions on Graphics, 25(3), ACM press, pp. 614-623.

Narzt, W., Pomberger, G., Ferscha, A., Kolb, D., Müller, R., Wieghardt, J., Hörtner, H. and Lindinger, C. (2004) A New Visualization Concept for Navigation Systems, in LNCS: User-Centered Interaction Paradigms for Universal Access in the Information Society, Springer.

Nash, E. B., Edwards, G. W., Thompson, J. A. and Barfield, W. (2000) A Review of Presence and Performance in Virtual Environments, in: International Journal of Human Computer Interaction, 12(1), Taylor \& Francis, pp. 1-41.

Omer, I., Golblatt, R., Talmor, K. and Roz, A. (2006) Enhancing the Legibility of Virtual Cities by Means of Residents' Urban Image: A Wayfinding Support System, in: Complex Artificial Environments Simulation, Cognition and VR in the Study and Planning of Cities, Springer.

Palmer, S.E. (1978) Fundamental Aspects of Cognitive Representation, in Rosch, E. and Lloyd, B.B.(eds.): Cognition and Categorization, Lawrence Erlbaum Assiociates, pp. 259-303.

Parish, Y.I.H. and Müller, P. (2001) Procedural Modeling of Cities, in: SIGGRAPH'01: Proceedings of the 28th Annual Conference on Computer Graphics and Interactive Techniques, ACM press, pp. 301-308.

Peters, D. and Richter, K.-F. (2008) Taking off to the Third Dimension - Schematization of Virtual Environments in: International Journal of Spatial Data In- 
frastructures Research, Vol 3, Joint Research Centre of the European Commission.

Richardson, A. R., Montello, D. R. and Hegarty, M. (1999) Spatial Knowledge Acquisition from Maps and From Navigation in Real and Virtual Environments, in: Memory and Cognition, 27, Psychonomic Society, pp. 741-750.

Riecke, B. E. (2003) How Far Can We Get With Just Visual Information? Path Integration and Spatial Updating Studies in Virtual Reality, $\mathrm{PhD}$ thesis, Universität Tübingen, Fakultät für Mathematik und Physik.

Strube, G. (1992) Contemporay Knowledge Engineering and Cognition, in: Schmalhofer, F., Strube, G., and Wetter, T.(eds): The Role of Cognitive Science in Knowledge Engineering, Springer, pp. 161-174.

Trapp, M., Glander, T., Buchholz, H. and Döllner, J. (2008) 3D Generalization Lenses for Interactive Focus + Context Visualization of Virtual City Models, in Proceedings of the 12th International Conference on IEEE Information Visualization, IEEE Computer Society Press, pp. 356-361.

Tversky, B., and Lee, P. U. (1999) Pictorial and Verbal Tools for Conveying Routes, in Freksa, C. and Mark, D.M. (eds.): LNCS: Spatial Information Theory. Cognitive and computational foundations of geographic information science.pp. 51-64.

Wein, R., Fogel, E., Zukerman, B. and Halperin, D. (2007) 2D Arrangements, in CGAL Editorial Board (ed.): CGAL User and Reference Manual. 3.3 edition. 